\title{
Patient, study thyself
}

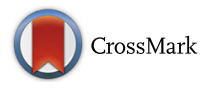

\author{
Paul Wicks
}

\begin{abstract}
The past 15 years have seen the emergence of a new paradigm in medical research, namely of people living with medical conditions (whether patients, parents, or caregivers) using digital tools to conduct N-of-1 trials and scientifically grounded research on themselves, whilst using the Internet to form communities of like-minded individuals willing to self-experiment. Prominent examples can be found in amyotrophic lateral sclerosis/motor neurone disease (the 'lithium study' on PatientsLikeMe), Parkinson's disease ('digital patient' Sara Riggare), and diabetes (the 'open artificial pancreas' of the \#WeAreNotWaiting movement). Through transparency, data sharing, open source code, and publication in the peer-reviewed scientific literature, such activities conform to expected scientific conventions. However, other conventions, such as ethical oversight, regulation, professionalization, and the ability to translate this new form of relatively biased data into generalizable decisions, remain challenged. While critics worry such participant-led research merely muddies the waters of high-quality medical research and exposes patients to new harms, the potential is there to enroll millions of active minds in unravelling the wicked problems of complex medical disorders that degrade the human health span.
\end{abstract}

Keywords: Patient engagement, Medical informatics, Patient-reported outcomes, Smartphones, Machine learning

\section{Background}

At the time of BMC Medicine's launch 15 years ago, I was conducting what I thought was fairly 'high-tech' neuropsychology research for my $\mathrm{PhD}$. Each week, I would drive out to the homes of people living with amyotrophic lateral sclerosis (ALS) to administer computerized psychological tests and invite them to our neuroimaging unit to have MRI and PET brain scans [1]. Since then, the most visible changes in medical research have been technological; for example, the heavy 486 laptops and the stack of inch-thick paper test batteries that I wheeled around in a $22 \mathrm{~kg}$ suitcase would now work more reliably as software apps on a $0.5 \mathrm{~kg}$ iPad [2]. However, I do not believe that the consumer electronics revolution of smartphones, tablets, and social media is the most profound change to have taken place during that time.

\section{Patients are doing it for themselves}

Instead, I think the largest shift in the past 15 years has been social, wherein patients, caregivers, parents, and family members have realized that 'research' is not just something that professionals do to them when they venture forth from their ivory towers, but that science and research are a set of tools and thinking methods that can be applied by anyone. My own wakeup call arrived in 2002, when I volunteered to take over as webmaster for an online community of people with ALS in the UK, called BUILD-UK [3]. As I was studying the supposedly rare issue of dementia in ALS, I was relentlessly grilled by patients rightfully asking why I was studying a potentially rare consequence of the disease that would take years to reap insights when patients were dying right now. Could the money not be better deployed towards clinical trials? I was surprised and impressed at the way they dissected clinical trial protocols, posed many of the same research questions I wondered about at conferences, and actually generated solutions that could immediately help other patients like them, rather than waiting for a peer-reviewed article to appear in print several years later. Most research professionals I spoke to seemed to merely tolerate 'lay people' having these discussions. However, not until one patient requested knowledge of their trial group allocation and actually had their study medication sent to a lab for testing, were they taken seriously [4].

Correspondence: pwicks@patientslikeme.com

PatientsLikeMe, 160 2nd Street, Cambridge, MA 02142, USA

(c) The Author(s). 2018 Open Access This article is distributed under the terms of the Creative Commons Attribution 4.0 International License (http://creativecommons.org/licenses/by/4.0/), which permits unrestricted use, distribution, and reproduction in any medium, provided you give appropriate credit to the original author(s) and the source, provide a link to the Creative Commons license, and indicate if changes were made. The Creative Commons Public Domain Dedication waiver (http://creativecommons.org/publicdomain/zero/1.0/) applies to the data made available in this article, unless otherwise stated. 


\section{Participant-led clinical trials and trial unblinding in ALS}

In early 2008, another group of exasperated ALS patients initiated their own participant-led trial of the drug lithium carbonate, which had been touted by some over-enthusiastic professional researchers as a near-cure for this dreadful disease [5]. Within 6 months, a patient in Brazil and a caregiver in the United States had rallied over 160 patients (10 times the original paper's sample size) to begin taking the drug off-label whilst systematically reporting their dosage, patient reported outcomes, side effects, and weight as well as running their own statistical analyses publicly on a weekly basis. All the data was reported online so that participants, and other members of the community, could see the data coming through in real-time. Some patients even recorded pre-/ post-exposure videos or tried to conduct objective tests of muscle strength. Building on internal analyses by the patients, the research team at PatientsLikeMe shared our own analysis at the International ALS/Motor Neurone Disease Symposium just 9 months later, wherein we found that lithium did not work [6]; we later shared our data for others to check [7]. At least five subsequent randomized control trials by academic researchers confirmed these findings [8]. However, critics of the participant-led study pointed to the lack of a traditional protocol, lack of blinding, and questionable ethics [9]. When a later tranche of the ALS community used the same approach to begin attempting to systematically unblind themselves at scale in blinded randomized control trials, alarm bells really started ringing [10]. Regardless of what researchers thought, patients were making their feelings clear - they did not have the time to wait for answers. I knew that to be true since, tragically, all participants in my PhD study had died by the time the results were formally published and it is only today that the implications are making their way into clinical practice [11].

\section{Who can grant you permission to experiment on yourself?}

When Swedish engineer Sara Riggare was diagnosed with Parkinson's disease aged 32, she did not just accept her doctor's advice - after all, she only saw him for approximately 1 hour annually and was responsible for her own self-care for the remaining 8765 hours. She enrolled herself for a PhD in Parkinson's disease at the Karolinska Institute and put her training to use. Sara devised objective and quantitative measures of how her dopaminergic system was functioning and interacting with her medications using smartphone technology to measure her finger tapping speed [12]. She has published peer-reviewed studies, including a survey of Swedish Parkinson's patients on how they self-manage [13] and an N-of-1 study using nicotine through an electronic cigarette to modulate symptoms and side effects [14]. Sara's pioneering work is interesting enough that she has even been a featured 'exhibit' at the British Science Museum and a celebrated inspiration to patients and researchers alike; yet, 6 years after starting her doctoral program, she hit an unexpected roadblock. Because she had neither sought nor been granted ethical approval to experiment on herself, the doctoral dissertation committee declined her application to defend her $\mathrm{PhD}$ thesis (http://www.riggare.se/ 2018/09/16/ethics-and-phd/\#more-1979). In practice, her studies had merely involved tapping her smartphone over a few days and smoking an e-cigarette, both of her own volition. Whereas ethical standards were constructed on a national level and centered around medical and educational institutions, the self-studying patient violated many of these assumptions. In one study, Sara tested her tapping speed while on an airplane crossing international waters could Swedish law still apply? Such 'edge cases' are essential in adapting our existing frameworks while being cautious not to erode key protections for potentially vulnerable people [15].

\section{Hacking diabetes}

However, participant-led research does not just end with a published peer-reviewed journal article. In 2014, a ground-breaking initiative was led by people living with diabetes in conjunction with parents of children living with the condition. They united under the hashtag \#WeAreNotWaiting and used their technical abilities to construct new 'open loop' systems - out of jury-rigged configurations - in their continuous glucose monitors, s-martphones, wearable devices, and older insulin pumps hacked to receive instructions from crowd-sourced software code [16]. While early results have been positive, including a high degree of safety and an impressive effect on HbA1c levels among this self-selecting group, the regulatory issues have been understandably complex [17]. The US Food and Drug Administration has engaged with the Nightscout group and so long as patients are building their own systems, rather than distributing them for commercial use, they appear exempt from regulation [17]. While it might be safer for an experienced builder to construct a closed loop system for another patient, this would bring with it unbearable liability and risk [18]. Thus, worldwide, small teams of people meet for 'DIY build parties' to discuss checklists and construct their own open loop systems. Once online, people share their experiences, data, and code to facilitate others to participate (https:// www.diabettech.com/).

\section{Conclusions}

The history of medicine encompasses much praise for doctors and scientists who experimented on themselves 
- Dr. Werner Forssmann won the Nobel prize after performing the first heart catheterization procedure on himself, and Dr. Barry Marshall won the prize for giving himself a stomach ulcer by swallowing a petri dish full of bacteria (https://scienceofparkinsons.com/2017/10/ 30/one/\#more-47943). Perhaps it is not so farfetched to think that in the next 15 years we might see a patient with no formal scientific or medical training win a Nobel prize for insights that arose from studying themselves and their peers. Perhaps one day the mantra will be "Patient, cure thyself".

\section{Acknowledgements}

The author is grateful to the patients and caregivers who have shared their experiences and participated in research.

\section{Funding}

No funding.

\section{Author's contributions}

PW drafted, revised, and finalized the manuscript. The author read and approved the final manuscript.

\section{Author's information}

PW is a researcher, patient, and caregiver. PW serves as Vice President of Innovation at PatientsLikeMe, an online community for people living with medical conditions. Specializing in the conduct of clinical research using the Internet, PW is responsible for shaping the scientific validity of the PatientsLikeMe platform and generating insights from personal health data shared by members. This sharing of online medical data has led to over 100 studies including a patient-driven observational trial of lithium in ALS, digital tools to develop patient-reported outcome measures, a 'dose-response' curve for the benefits of friendship between patients, and methods for patient-centered and virtual clinical trial designs. He sits on the editorial board of several journals, including BMC Medicine, the BMJ, and The Patient. Prior to joining PatientsLikeMe, PW worked at the Institute of Psychiatry (King's College London) studying cognition and neuroimaging in rare forms of ALS, and the psychological consequences of Parkinson's disease.

\section{Competing interests}

PW is an employee of PatientsLikeMe (which facilitates patient-led research and self-experimentation) and holds stock options in the company. PW is an associate editor at the Journal of Medical Internet Research and is on the Editorial Boards of the BMJ, BMC Medicine, and Digital Biomarkers. The PatientsLikeMe Research Team has received research funding (including conference support and consulting fees) from Abbvie, Accorda, Actelion, Alexion, Amgen, AstraZeneca, Avanir, Biogen, Boehringer Ingelheim, Celgene, EMD, Genentech, Genzyme, Janssen, Johnson \& Johnson, Merck, Neuraltus, Novartis, Otsuka, Permobil, Pfizer, Sanofi, Shire, Takeda, Teva, and UCB. The PatientsLikeMe R\&D team has received research grant funding from Kaiser Permanente, the Robert Wood Johnson Foundation, Sage Bionetworks, The AKU Society, and the University of Maryland. PW has received speaker fees from Bayer and honoraria from Roche, ARISLA, AMIA, IMI, PSI, and the BMJ.

\section{Publisher's Note}

Springer Nature remains neutral with regard to jurisdictional claims in published maps and institutional affiliations.

Received: 25 October 2018 Accepted: 12 November 2018 Published online: 23 November 2018

\section{References}

1. Wicks P, Turner MR, Abrahams S, Hammers A, Brooks DJ, Leigh PN, et al. Neuronal loss associated with cognitive performance in amyotrophic lateral sclerosis: an (11C)-flumazenil PET study. Amyotrophic Lateral Scler. 2008;9: 43-9.
2. Germine L, Nakayama K, Duchaine BC, Chabris CF, Chatterjee G, Wilmer JB. Is the Web as good as the lab? Comparable performance from Web and lab in cognitive/perceptual experiments. Psychon Bull Rev. 2012;19:847-57.

3. Down K, Sinha A, Hughes R. Building user participation to reshape services for people affected by motor neurone disease. J Interprof Care. 2002;16(3): 289-90.

4. Wicks P. Clinical trial blinding in the age of social media. Blinding as a solution to bias. New York: Elsevier; 2016. p. 97-106.

5. Fornai F, Longone P, Cafaro L, Kastsiuchenka O, Ferrucci M, Manca ML, et al. Lithium delays progression of amyotrophic lateral sclerosis. Proc. Natl. Acad. Sci. U.S.A. 2008;105:2052-7.

6. Wicks P, Massagli M, Frost J, Macedo H, Felzer KA. patient-led trial of lithium in ALS using the internet. Amyotrophic lateral sclerosis. ALS. 2008;9:S59.

7. Wicks P, Vaughan TE, Massagli MP, Heywood J. Accelerated clinical discovery using self-reported patient data collected online and a patientmatching algorithm. Nat. Biotechnol. 2011;29:411-4.

8. Armon C. Is the lithium-for-ALS genie back in the bottle? Not quite. Neurology. 2010;75:586-7.

9. Stefanou M-I, Amygdalos I. Patient-led research in amyotrophic lateral sclerosis: Quo vadis? Amyotroph Lateral Scler Frontotemporal Degener. 2015;16:418-22.

10. Wicks $P$, Vaughan $T$, Heywood J. Subjects no more: what happens when trial participants realize they hold the power? BMJ. 2014;348:g368.

11. Wicks P, Albert SM. It's time to stop saying 'the mind is unaffected' in ALS. Neurology. 2018;:10.1212/WNL.0000000000006303.

12. Riggare S, Hägglund M. Precision Medicine in Parkinson's Disease Exploring Patient-Initiated Self-Tracking. J Parkinsons Dis. 2018;8:441-6.

13. Riggare S, Höglund PJ, Hvitfeldt Forsberg H, Eftimovska E, Svenningsson P, Hägglund M. Patients are doing it for themselves: A survey on diseasespecific knowledge acquisition among people with Parkinson's disease in Sweden. Health Informatics J; 2017;2013:1460458217704248.

14. Riggare S, Unruh KT, Sturr J, Domingos J, Stamford JA, Svenningsson P, et al. Patient-driven N-of-1 in Parkinson's Disease. Lessons Learned from a Placebo-controlled Study of the Effect of Nicotine on Dyskinesia. Methods Inf Med. 2017:56:e123-8.

15. Steuli JC, Vayena E. The promising revolution of participant-led research in rare neurological diseases; Potential benefits and pitfalls. Epileptologie. 2016; 32:177-82.

16. Lewis D, Leibrand S, \#OpenAPS Community. Real-World Use of Open Source Artificial Pancreas Systems. J Diabetes Sci Technol; 2016;10:1411-1.

17. Lee JM, Hirschfeld E, Wedding J. A Patient-Designed Do-lt-Yourself Mobile Technology System for Diabetes: Promise and Challenges for a New Era in Medicine. JAMA. 2016;315:1447-8.

18. Omer T. Empowered citizen "health hackers" who are not waiting. BMC Med. 2016;14:118. 